\title{
A note on the Poincaré inequality
}

\author{
by \\ Alireza Ranjbar-Motlagh (Tehran)
}

\begin{abstract}
The Poincaré inequality is extended to uniformly doubling metric-measure spaces which satisfy a version of the triangle comparison property. The proof is based on a generalization of the change of variables formula.
\end{abstract}

1. Introduction. The purpose of this paper is to prove the Poincaré type inequality for metric-measure spaces, that is, metric spaces $(X, d)$ with a measure $\mu$ (see [EG], [He] and [R] for the basic definitions). It is wellknown that the Poincaré inequality implies the Sobolev and isoperimetric inequalities in doubling and smooth spaces; see for instance $[\mathrm{HK}]$ and $[\mathrm{F}]$.

Heinonen and Koskela $[\mathrm{HeK}]$ introduced the concept of upper gradient and they proved the Poincaré type inequality for abstract spaces. Bourdon and Pajot $[\mathrm{BP}]$ showed that there exist metrics on the boundary of some hyperbolic buildings such that the Poincaré type inequality is valid and the space is Ahlfors regular of non-integer dimension. Also, Laakso [L] constructed Ahlfors regular spaces of any given dimension greater than one such that the Poincaré type inequality is valid. Hanson and Heinonen $[\mathrm{HH}]$ constructed, for any integer $n \geq 2$, a space with topological dimension $n$ and Ahlfors regular of dimension $n$ which supports the Poincaré type inequality, but has no manifold point. Jerison [J], Garofalo and Nheiu [GN], and Lanconelli and Morbidelli [LM] obtained the Poincaré type inequality for Carnot-Carathéodory spaces. Semmes [Se] proved the Poincaré type inequality for spaces which have "nice" families of curves. Moreover, Cheeger $[\mathrm{Ch}]$ provided applications of the Poincaré type inequality for abstract spaces. For more works about this topic on Riemannian manifolds, groups and graphs see $[\mathrm{B}],[\mathrm{Li}]$ and $[\mathrm{VSC}]$. See also $[\mathrm{HK}]$ and the references therein.

2000 Mathematics Subject Classification: Primary 26D10, 46E35; Secondary 46E30.

Key words and phrases: Poincaré inequality, generalized change of variables formula, Sobolev spaces.

The author was partially supported by FAPEMIG Grants CEX-946/98 and CEX00065/00 in Brazil, and IPM Grant 5/80/1140 in Iran. 
In this paper, we introduce a version of the triangle comparison property on metric spaces and a strong version of the doubling property on metricmeasure spaces such that the Poincaré inequality is valid in such spaces. Here, we use the definition of (extended) upper gradient on metric spaces in order for the Poincaré inequality (the norm of derivative) to make sense for abstract spaces. The proof is based on a generalization of the change of variables formula.

2. The basic definitions. In this section, we introduce a sufficient condition on metric-measure spaces which implies the Poincaré inequality. First, we recall some basic definitions relating to metric-measure spaces. For simplicity, we assume that all metric spaces are locally compact and all (outer) measures are Radon (see [EG] for definitions). We denote the closed ball of radius $R>0$ with center at $a$ by $B(a, R)$ (in a metric space $(X, d)$ ).

Definition 2.1. Let $(X, d, \mu)$ be a metric-measure space. We say that $X$ is a doubling space if there is $C_{0}>0$ such that

$$
\mu(B(x, 2 r)) \leq C_{0} \mu(B(x, r)) \quad \text { for all } x \in X \text { and } r>0 .
$$

A metric-measure space $(X, d, \mu)$ is called a uniformly doubling space if there is $C>0$ such that

$$
\mu(B(x, 2 r)) \leq C \mu(B(y, r)) \quad \text { for all } x, y \in X \text { and } r>0 .
$$

The constant $C$ is called the (uniform) doubling constant of $X$. It is clear that every uniformly doubling space is a doubling space.

Let $(X, d, \mu)$ be a metric-measure space. We say that $X$ is Ahlfors regular if there are $K>0$ and $n>0$ such that

$$
K^{-1} r^{n} \leq \mu(B(x, r)) \leq K r^{n} \quad \text { for all } x \in X \text { and } 0<r<\operatorname{diam}(X) .
$$

The number $n$ is called the dimension of $X$.

The uniformly doubling condition is weaker than Ahlfors regularity and stronger than the (usual) doubling condition.

Now, we introduce the spaces of bounded geometry; these spaces satisfy a version of the conclusion of the Rauch (Toponogov) comparison theorem for Riemannian manifolds.

Definition 2.2. Let $(X, d)$ be a metric space. We say that $X$ is a geodesic space if for every $x, y \in X$, there is a geodesic $\gamma_{x, y}:[0,1] \rightarrow X$ (with velocity $d(x, y)$ ) from $x$ to $y$, i.e. $d\left(\gamma_{x, y}(s), \gamma_{x, y}(t)\right)=|s-t| d(x, y)$, $\gamma_{x, y}(0)=x$ and $\gamma_{x, y}(1)=y$. Suppose that a function $\Phi: X \times X \times[0,1] \rightarrow X$ satisfies

$$
\Phi(x, y, s)=\Phi(y, x, 1-s)=\gamma_{x, y}(s)
$$

for $x, y \in X$ and $s \in[0,1]$, where $\gamma_{x, y}$ is a geodesic from $x$ to $y$. We say 
that the space $X$ has bounded geometry if there is $b>0$ such that for every $x, y, z \in X$, we have

$$
d(y, z) \leq b d(\Phi(x, y, t), \Phi(x, z, t)) \quad \text { for all } 1 / 2 \leq t \leq 1 .
$$

Next, we recall the definition of (extended) upper gradient which is a generalization of the norm of gradient for smooth functions.

Definition 2.3. Let $(X, d, \mu)$ be a metric-measure space. Let $\Phi$ (as in Definition 2.2) be a measurable mapping and let $u$ be a (real-valued) function on $X$. A non-negative Borel function $g$ is said to be an (extended) upper gradient for $u$ if

$$
|u(x)-u(y)| \leq d(x, y) \int_{0}^{1} g(\Phi(x, y, s)) d s \quad \text { for a.e. }(x, y) \in X \times X .
$$

REMARK 2.4. The above definition of (extended) upper gradient is slightly weaker than [HeK, Def. 2.9]. Compare [Ch, Def. 2.8].

Proposition 2.5. Let $(X, d, \mu)$ denote the Euclidean space $\mathbb{R}^{n}$ with the usual Euclidean metric $d$ and arbitrary (Borel) measure $\mu$. Then:

(i) $X$ has bounded geometry.

(ii) $X$ is uniformly doubling if and only if it is Ahlfors regular of dimension $n$.

Proof. (i) This is obvious.

(ii) It is clear that every Ahlfors regular space is uniformly doubling. Now, suppose $(X, d, \mu)$ is uniformly doubling. Since $(X, d)$ is the usual Euclidean space, it is clear that for any ball $B$ of radius $r$, there are cubes $D$ and $D^{\prime}$ with diameters, respectively, $2 r$ and $2 \sqrt{2} r$, such that

$$
D \subset B \subset D^{\prime} .
$$

Therefore, without loss of generality, instead of balls we can consider cubes. Since every cube with diameter $s$ can be covered by $2^{m n}$ cubes with diameters $s / 2^{m}$, we obtain

$$
\frac{2^{m n}}{K} \mu\left(B\left(y, s / 2^{m}\right)\right) \leq \mu(B(x, s)) \leq 2^{m n} K \mu\left(B\left(y, s / 2^{m}\right)\right)
$$

for any $x, y \in X$ and positive number $s$, where $K$ is a constant which depends on the (uniform) doubling constant of $X$. Hence,

$$
\frac{\mu(B(z, s))}{s^{n}} \leq K \frac{\mu(B(w, r))}{r^{n}}
$$

for any $z, w \in X$ and positive numbers $r$ and $s$, where $K$ is a constant which depends on the doubling constant of $X$. 
EXAMPLE 2.6. Let $\left(M^{n}, g\right)$ be an $n$-dimensional Riemannian manifold whose sectional curvature is bounded, $\left|K_{M}\right| \leq K_{0}<\infty$, and whose injectivity radius is bounded from below, $\operatorname{inj}(M) \geq i_{0}>0$. Then every ball of radius less than $i_{0} / 4$ has bounded geometry as a metric space. This is a straightforward consequence of the Rauch comparison theorem. See for example [CE, Thm. 1.28].

Proposition 2.7. Let $(X, d, \mu)$ be a uniformly doubling metric-measure space which has bounded geometry. Let $\Phi$ (as in Definition 2.2) be a measurable mapping. Let $a \in X, R>0$ and $t \in[1 / 2,1]$. Then for every (measurable) subset $F \subset B(a, R)$, we have

$$
\mu(\{z \in B(a, R): \Phi(a, z, t) \in F\}) \leq M \mu(F),
$$

where $M$ is a constant which depends on $b$ and the (uniform) doubling constant of $X$.

Proof. Since $(X, d, \mu)$ is a uniformly doubling space which has bounded geometry, we have

$$
\mu(\{z \in B(a, R): \Phi(a, z, t) \in B(w, r)\}) \leq M \mu(B(w, r))
$$

for all $B(w, r) \subset B(a, R)$, where $M$ is a constant which depends on $b$ and the (uniform) doubling constant of $X$.

Let $F$ be a (measurable) subset of $B(a, R)$ and let $\delta>0$. By the Vitali covering lemma (see for example $[\mathrm{S}$, p. 9$]$ ), there is a sequence $\left\{B_{i}\right\}$ of mutually disjoint balls such that

$$
F \subset \bigcup_{i} \widehat{B}_{i} \text { and } \quad \mu\left(\bigcup_{i} \widehat{B}_{i} \backslash F\right) \leq \delta
$$

where $\widehat{B}_{i}$ denotes the ball whose center is the same as $B_{i}$ and whose radius is 5 times the radius of $B_{i}$. Then, by $(2.1)$, we have

$$
\begin{aligned}
\mu(\{z \in B(a, R): \Phi(a, z, t) \in F\}) \leq \mu\left(\left\{z \in B(a, R): \Phi(a, z, t) \in \bigcup_{i} \widehat{B}_{i}\right\}\right) \\
\leq \mu\left(\bigcup_{i}\left\{z \in B(a, R): \Phi(a, z, t) \in \widehat{B}_{i}\right\}\right) \\
\leq \sum_{i} \mu\left(\left\{z \in B(a, R): \Phi(a, z, t) \in \widehat{B}_{i}\right\}\right) \\
\leq M \mu\left(\bigcup_{i} \widehat{B}_{i}\right) \leq M \sum_{i} \mu\left(\widehat{B}_{i}\right) \leq M \sum_{i} \mu\left(B_{i}\right) \\
=M \mu\left(\bigcup_{i} B_{i}\right) \leq M \mu\left(\bigcup_{i} \widehat{B}_{i}\right) .
\end{aligned}
$$


By choosing $\delta$ small enough, we have

$$
\mu(\{z \in B(a, R): \Phi(a, z, t) \in F\}) \leq M \mu(F) .
$$

Remark 2.8. In Proposition 2.7, if we assume that $\mu$ is an outer measure, we can remove the assumption of measurablity of $\Phi$ (and of $F$ ). See [EG] for the relevant definitions.

3. The main results. In this section, we show that the Poincare inequality is valid for uniformly doubling spaces which have bounded geometry.

We start with the following generalization of the change of variables formula.

Lemma 3.1 (Generalized change of variables). Let $(X, d, \mu)$ be a uniformly doubling metric-measure space which has bounded geometry. Let $g$ be a non-negative Borel measurable function on X. Let $\Phi$ (as in Definition 2.2) be a measurable mapping. Let $a \in X$ and $R>0$. Then, for a.e. $a \in X$, we have

$$
\int_{B(a, R)}\left(\int_{1 / 2}^{1} g(\Phi(a, y, s)) d s\right) d \mu(y) \leq M \int_{B(a, R)} g(z) d \mu(z),
$$

where $M$ is a constant which depends on $b$ and the (uniform) doubling constant of $X$.

Proof. Without loss of generality, we can assume that the mapping $h(y, s):=\Phi(a, y, s)$ is measurable. Let $\varepsilon$ be a small positive number. Since $X$ is a doubling space, we can construct a finite sequence $\left\{x_{i}\right\} \subset B(a, R)$ with the following properties:

$$
B\left(x_{i}, \varepsilon\right) \cap B\left(x_{j}, \varepsilon\right)=\emptyset \quad \text { for all } i \neq j, \quad B(a, R) \subset \bigcup_{i} B\left(x_{i}, 2 \varepsilon\right) .
$$

Then, by our assumptions,

$$
d\left(x_{i}, x_{j}\right) \leq b d\left(h\left(x_{i}, t\right), h\left(x_{j}, t\right)\right)
$$

for all $1 / 2 \leq t \leq 1$, and some $b>0$. Therefore, for every $1 / 2 \leq t \leq 1$, we get

$$
B\left(h\left(x_{i}, t\right), b^{-1} \varepsilon\right) \cap B\left(h\left(x_{j}, t\right), b^{-1} \varepsilon\right)=\emptyset
$$

for all $i \neq j$. Since $X$ is a uniformly doubling space, we obtain

$$
\sum_{i} \mu\left(B\left(x_{i}, 2 \varepsilon\right)\right) g\left(h\left(x_{i}, t\right)\right) \leq M \sum_{i} \mu\left(B\left(h\left(x_{i}, t\right), b^{-1} \varepsilon\right)\right) g\left(h\left(x_{i}, t\right)\right),
$$


where $M$ is a constant which depends on $b$ and the (uniform) doubling constant of $X$. It is enough to show that

$$
\int_{B(a, R)} g(h(y, t)) d \mu(y) \leq M \int_{B(a, R+2 \varepsilon)} g(z) d \mu(z)
$$

for all $1 / 2 \leq t \leq 1$ and $\varepsilon>0$, where $M$ is a constant which depends on $b$ and the (uniform) doubling constant of $X$.

The proof is based on the following statements:

(a) If (3.4) is valid for bounded measurable functions $g$, then it is valid for all measurable functions $g$.

(b) If both $g(\cdot)$ and $g(h(\cdot, t))$ are continuous, then (3.4) is valid.

(c) If (3.4) is valid for $g$ continuous, then it is valid for $g$ measurable.

(d) (3.4) is valid for $g$ continuous.

Proof of (a). Suppose that (3.4) holds for all bounded functions. Let $g$ be any measurable function. Apply it to the bounded functions

$$
g_{N}(x):=g(x) \chi_{\{y: g(y) \leq N\}}(x),
$$

where $\chi$ denotes the characteristic function and $N$ is a positive integer. Letting $N \rightarrow \infty$ implies that (3.4) is valid for $g$.

From now on, we assume that $0 \leq g(x) \leq K$ for all $x$ and some number $K$.

Proof of (b). This is an immediate consequence of (3.3). Note that we have assumed that all metric spaces are locally compact, therefore, every continuous function on a closed (and bounded) ball is uniformly continuous. Also, it is easy to see that a doubling space is complete iff every closed (and bounded) ball is compact.

Proof of (c). Suppose that (3.4) holds for all continuous functions and let $g$ be measurable. Let $\delta>0$. By the Luzin theorem (see [Ru, p. 55]) there is a continuous function $u$ such that

$$
0 \leq u(x) \leq K, \quad \forall x, \quad \text { and } \quad \mu(A) \leq \delta,
$$

where $A:=\{x \in B(a, R): u(x) \neq g(x)\}$. By Proposition 2.7, we have

$$
\mu(\{y \in B(a, R): u(h(y, t)) \neq g(h(y, t))\}) \leq M \delta,
$$

where $M$ is a constant which depends on $b$ and the (uniform) doubling constant of $X(1 / 2 \leq t \leq 1$ is a fixed number). Then, since (3.4) holds for $u$, we have

$$
-2 K(M \delta)+\int_{B(a, R)} g(h(y, t)) d \mu(y) \leq M\left(\int_{B(a, R)} g(z) d \mu(z)+K \delta\right) .
$$

Letting $\delta \rightarrow 0$ implies that $g$ satisfies (3.4). 
Proof of $(\mathrm{d})$. Let $g$ be continuous and $\delta>0$. By the Luzin theorem there is a continuous function $v$ such that

$$
0 \leq v(x) \leq K, \quad \forall x, \quad \text { and } \quad \mu(S) \leq \delta,
$$

where $S:=\{y \in B(a, R): v(y) \neq g(h(y, t))\}$. Put $U:=B(a, R) \backslash S$. Let $\varepsilon$ be a small positive number. We can choose a finite sequence $\left\{x_{i}\right\} \subset B(a, R)$ satisfying the following conditions:

$$
B\left(x_{i}, \varepsilon\right) \cap B\left(x_{j}, \varepsilon\right)=\emptyset \quad \text { for all } i \neq j, \quad B(a, R) \subset \bigcup_{i} B\left(x_{i}, 4 \varepsilon\right),
$$

and

$$
x_{j} \in U \quad \text { or } \quad B\left(x_{j}, \varepsilon\right) \cap U=\emptyset
$$

for all $j$. To do this, we choose a finite sequence $\left\{z_{i}\right\} \subset U$ such that

$$
B\left(z_{i}, \varepsilon\right) \cap B\left(z_{j}, \varepsilon\right)=\emptyset \quad \text { for all } i \neq j, \quad U \subset \bigcup_{i} B\left(z_{i}, 2 \varepsilon\right) .
$$

Moreover, we choose a finite sequence $\left\{w_{i}\right\} \subset V:=B(a, R) \backslash \bigcup_{i} B\left(z_{i}, 4 \varepsilon\right)$ such that (if $V \neq \emptyset$ )

$$
B\left(w_{i}, \varepsilon\right) \cap B\left(w_{j}, \varepsilon\right)=\emptyset \quad \text { for all } i \neq j, \quad V \subset \bigcup_{i} B\left(w_{i}, 2 \varepsilon\right) .
$$

Then the sequence $\left\{x_{i}\right\}=\left\{z_{i}\right\} \cup\left\{w_{i}\right\}$ is as required. Note that $\left\{x_{i}\right\}$ satisfies (3.2) and (3.3). We have

$$
\int_{B(a, R)} v(y) d \mu(y) \leq \sum_{i} \int_{B\left(x_{i}, 4 \varepsilon\right)} v(y) d \mu(y) \leq \sum_{i} \mu\left(B\left(x_{i}, 4 \varepsilon\right)\right) \sup _{B\left(x_{i}, 4 \varepsilon\right)} v
$$

Also,

$$
\begin{aligned}
\sum_{i} \mu\left(B\left(h\left(x_{i}, t\right), b^{-1} \varepsilon\right)\right) \inf _{B\left(h\left(x_{i}, t\right), b^{-1} \varepsilon\right)} g & \leq \sum_{i} \int_{B\left(h\left(x_{i}, t\right), b^{-1} \varepsilon\right)} g(z) d \mu(z) \\
& \leq \int_{B\left(a, R+b^{-1} \varepsilon\right)} g(z) d \mu(z) .
\end{aligned}
$$

Now, since $g$ and $v$ are uniformly continuous on closed (and bounded) balls (and using inequality (3.3)), we have

$$
\begin{aligned}
\sum_{i} \mu\left(B\left(x_{i}, 4 \varepsilon\right)\right) \sup _{B\left(x_{i}, 4 \varepsilon\right)} v & \\
& \leq \sum_{x_{i} \in U} \mu\left(B\left(x_{i}, 4 \varepsilon\right)\right) \sup _{B\left(x_{i}, 4 \varepsilon\right)} v+\sum_{x_{i} \notin U} \mu\left(B\left(x_{i}, 4 \varepsilon\right)\right) \sup _{B\left(x_{i}, 4 \varepsilon\right)} v \\
& \leq M \sum_{x_{i} \in U} \mu\left(B\left(x_{i}, \varepsilon\right)\right) \sup _{B\left(x_{i}, 4 \varepsilon\right)} v+M \sum_{x_{i} \notin U} \mu\left(B\left(x_{i}, \varepsilon\right)\right) \sup _{B\left(x_{i}, 4 \varepsilon\right)} v \\
& \leq M\left[\sum_{x_{i} \in U} \mu\left(B\left(h\left(x_{i}, t\right), b^{-1} \varepsilon\right)\right)\left[v\left(x_{i}\right)+\eta\right]\right]+M \sum_{x_{i} \notin U} \mu\left(B\left(x_{i}, \varepsilon\right)\right) K
\end{aligned}
$$




$$
\begin{aligned}
\leq & M \sum_{x_{i} \in U} \mu\left(B\left(h\left(x_{i}, t\right), b^{-1} \varepsilon\right)\right) v\left(x_{i}\right) \\
& +M \sum_{x_{i} \in U} \mu\left(B\left(h\left(x_{i}, t\right), b^{-1} \varepsilon\right)\right) \eta+M K \mu(S) \\
\leq & \eta+M\left[\sum_{x_{i} \in U} \mu\left(B\left(h\left(x_{i}, t\right), b^{-1} \varepsilon\right)\right) v\left(x_{i}\right)\right]+M K \mu(S) \\
\leq & \eta+M\left[\sum_{x_{i} \in U} \mu\left(B\left(h\left(x_{i}, t\right), b^{-1} \varepsilon\right)\right) g\left(h\left(x_{i}, t\right)\right)\right]+M K \delta,
\end{aligned}
$$

where $\eta \geq 0$ depends on $g, v, B(a, R)$ and $M$. Moreover, $\eta \rightarrow 0$ as $\varepsilon \rightarrow 0$. Therefore,

$$
\begin{aligned}
& \int_{B(a, R)} v(y) d \mu(y) \\
& \leq \eta+M\left[\sum_{x_{i} \in U} \mu\left(B\left(h\left(x_{i}, t\right), b^{-1} \varepsilon\right)\right) g\left(h\left(x_{i}, t\right)\right)\right]+M K \delta \\
& \leq \eta+M\left[\sum_{x_{i} \in U} \mu\left(B\left(h\left(x_{i}, t\right), b^{-1} \varepsilon\right)\right)\left[\inf _{B\left(h\left(x_{i}, t\right), b^{-1} \varepsilon\right)} g+\eta\right]\right]+M K \delta \\
& \leq \eta+M\left[\sum_{x_{i} \in U} \mu\left(B\left(h\left(x_{i}, t\right), b^{-1} \varepsilon\right)\right) \inf _{B\left(h\left(x_{i}, t\right), b^{-1} \varepsilon\right)} g\right] \\
& +M\left[\sum_{x_{i} \in U} \mu\left(B\left(h\left(x_{i}, t\right), b^{-1} \varepsilon\right)\right) \eta\right]+M K \delta \\
& \leq \eta+M\left[\sum_{i} \mu\left(B\left(h\left(x_{i}, t\right), b^{-1} \varepsilon\right)\right) \inf _{B\left(h\left(x_{i}, t\right), b^{-1} \varepsilon\right)} g\right]+M K \delta \\
& \leq \eta+M\left[\int_{B\left(a, R+b^{-1} \varepsilon\right)} g(z) d \mu(z)\right]+M K \delta .
\end{aligned}
$$

This implies that

$$
\begin{aligned}
& \int_{B(a, R)} g(h(y, t)) d \mu(y)=\int_{S} g(h(y, t)) d \mu(y)+\int_{U} g(h(y, t)) d \mu(y) \\
& =\int_{S} g(h(y, t)) d \mu(y)+\int_{U} v(y) d \mu(y) \leq K \mu(S)+\int_{B(a, R)} v(y) d \mu(y) \\
& \leq K \delta+\int_{B(a, R)} v(y) d \mu(y) \leq \eta+M K \delta+M \int_{B\left(a, R+b^{-1} \varepsilon\right)} g(z) d \mu(z) .
\end{aligned}
$$


Letting $\varepsilon \rightarrow 0$, we obtain

$$
\int_{B(a, R)} g(h(y, t)) d \mu(y) \leq M K \delta+M \int_{B(a, R)} g(z) d \mu(z),
$$

and letting $\delta \rightarrow 0$ yields

$$
\int_{B(a, R)} g(h(y, t)) d \mu(y) \leq M \int_{B(a, R)} g(z) d \mu(z)
$$

for all $1 / 2 \leq t \leq 1$

REMARK 3.2. In a quite general setting, the mapping $\Phi$ is (can be chosen) measurable. In fact, by a construction due to Kuratowski and RyllNardzewski [AC, p. 90], we can construct a measurable selection.

Theorem 3.3 (Weak Poincaré inequality). Let $(X, d, \mu)$ be a uniformly doubling metric-measure space which has bounded geometry. Let $\Phi$ (as in Definition 2.2) be a measurable mapping. Let $g$ be an (extended) upper gradient for a measurable function $u$ on $X$. Then

$$
f_{B(a, R)} f_{B(a, R)}|u(x)-u(y)| d \mu(x) d \mu(y) \leq M R \underset{B(a, 3 R)}{f} g(z) d \mu(z),
$$

where $a \in X, R>0$, and $M$ is a constant which depends on $b$ and the (uniform) doubling constant of $X$.

Proof. By the definition of (extended) upper gradient, we have

$$
|u(x)-u(y)| \leq d(x, y) \int_{0}^{1} g(\Phi(x, y, s)) d s
$$

for a.e. $(x, y) \in X \times X$, where $\Phi(x, y, s)$ is as in Definition 2.2. By integrating, we have

$$
\begin{aligned}
\underset{B(a, R) B(a, R)}{f} \mid u(x)- & u(y) \mid d \mu(x) d \mu(y) \\
\leq & 2 R f_{B(a, R)} f_{B(a, R)} \int_{0}^{1} g(\Phi(x, y, s)) d s d \mu(x) d \mu(y) \\
= & 2 R\left(\underset{B(a, R) B(a, R)}{f} \int_{B(a)}^{1 / 2} g(\Phi(x, y, s)) d s d \mu(x) d \mu(y)\right. \\
& \left.+\underset{B(a, R) B(a, R) 1 / 2}{f} \int_{f(x, 2)}^{1} g(\Phi(x, y, s)) d s d \mu(x) d \mu(y)\right)
\end{aligned}
$$




$$
\leq 4 R \underset{B(a, R)}{f} f_{B(a, R)} \int_{1 / 2}^{1} g(\Phi(x, y, s)) d s d \mu(y) d \mu(x) .
$$

Hence,

$$
\begin{aligned}
& \quad \underset{B(a, R) B(a, R)}{f}|u(x)-u(y)| d \mu(x) d \mu(y) \\
& \leq K R f_{B(a, R)}\left(f_{B(x, 2 R)} \int_{1 / 2}^{1} g(\Phi(x, y, s)) d s d \mu(y)\right) d \mu(x)
\end{aligned}
$$

for some constant $K$ which depends on the doubling constant of $X$. Then, by the generalized change of variables formula,

$$
\begin{aligned}
& f_{B(a, R)} f_{B(a, R)}|u(x)-u(y)| d \mu(x) d \mu(y) \\
& \quad \leq M R f_{B(a, R)}\left(f_{B(x, 2 R)} g(z) d \mu(z)\right) d \mu(x) \leq M R f_{B(a, 3 R)} g(z) d \mu(z)
\end{aligned}
$$

for some constant $M$ which depends on $b$ and the (uniform) doubling constant of $X$.

REMARK 3.4. Under the notations and assumptions of Theorem 3.3, if the ball $B(a, R)$ is convex, then

$$
f_{B(a, R)} f_{B(a, R)}|u(x)-u(y)| d \mu(x) d \mu(y) \leq M R f_{B(a, R)} g(z) d \mu(z) .
$$

\section{References}

[A] R. A. Adams, Sobolev Spaces, Academic Press, New York, 1975.

[AC] J. P. Aubin and A. Cellina, Differential Inclusions, Grundlehren Math. Wiss. 264, Springer, New York, 1984.

[BP] M. Bourdon and H. Pajot, Poincaré inequalities and quasiconformal structure on the boundary of some hyperbolic buildings, Proc. Amer. Math. Soc. 127 (1999), 2315-2324.

[B] P. Buser, A note on the isoperimetric constant, Ann. Sci. École Norm. Sup. 15 (1982), 213-230.

[Ch] J. Cheeger, Differentiability of Lipschitz functions on metric measure spaces, Geom. Funct. Anal. 9 (1999), 428-517.

[CE] J. Cheeger and D. Ebin, Comparison Theorems in Riemannian Geometry, NorthHolland, Amsterdam, 1975.

[EG] L. C. Evans and R. F. Gariepy, Measure Theory and Fine Properties of Functions, Stud. Adv. Math., CRC Press, Boca Raton, FL, 1992.

[F] H. Federer, Geometric Measure Theory, Grundlehren Math. Wiss. 153, Springer, New York, 1969. 
[GN] N. Garofalo and D.-M. Nhieu, Isoperimetric and Sobolev inequalities for CarnotCarathéodory spaces and existence of minimal surfaces, Comm. Pure Appl. Math. 49 (1996), 1081-1144.

[HH] B. Hanson and J. Heinonen, An n-dimensional space that admits a Poincaré inequality but has no manifold points, Proc. Amer. Math. Soc. 128 (2000), 33793390.

[HK] P. Hajłasz and P. Koskela, Sobolev met Poincaré, Mem. Amer. Math. Soc. 688 (2000).

[He] J. Heinonen, Lectures on Analysis on Metric Spaces, Springer, New York, 2001.

$[\mathrm{HeK}] \quad$ J. Heinonen and P. Koskela, Quasiconformal maps in metric spaces with controlled geometry, Acta Math. 181 (1998), 1-61.

[J] D. Jerison, The Poincaré inequality for vector fields satisfying Hörmander's condition, Duke Math. J. 53 (1986), 503-523.

[L] T. J. Laakso, Ahlfors Q-regular spaces with arbitrary $Q>1$ admitting weak Poincaré inequality, Geom. Funct. Anal. 10 (2000), 111-123.

[LM] E. Lanconelli and D. Morbidelli, On the Poincaré inequality for vector fields, Ark. Mat. 38 (2000), 327-342.

[Li] P. Li, Lecture Notes on Geometric Analysis, Lecture Notes Ser. 6, Seoul National Univ., Research Institute of Mathematics, Global Analysis Research Center, Seoul, 1993.

[R] A. Ranjbar-Motlagh, Analysis on metric-measure spaces, Ph.D. thesis, New York Univ., 1998.

[Ru] W. Rudin, Real and Complex Analysis, 3rd ed., McGraw-Hill, 1987.

[Se] S. Semmes, Finding curves on general spaces through quantitative topology, with applications to Sobolev and Poincaré inequalities, Selecta Math. 2 (1996), 155295.

[S] E. M. Stein, Singular Integrals and Differentiability Properties of Functions, Princeton Univ. Press, 1970.

[VSC] N. T. Varopoulos, L. Saloff-Coste and T. Coulhon, Analysis and Geometry on Groups, Cambridge Univ. Press, Cambridge, 1992.

Department of Mathematical Sciences

Sharif University of Technology

P.O. Box 11365-9415

Tehran, Iran

E-mail: ranjbarm@sharif.edu

Received October 31, 2000

Revised version February 25, 2002 